\title{
Validación de un prototipo de sistema captación de agua de lluvia para uso doméstico y consumo humano
}

\author{
Validation of a prototype of rainwater harvesting system for domestic use and human \\ consumption
}

\author{
Juan Uriel Avelar Roblero ${ }^{*}$, Juan Rafael Sánchez Bravo², Abraham Domínguez Acevedo², \\ Claudio Lobato de La Cruz², Oscar Raúl Mancilla Villa ${ }^{3}$
}

\begin{abstract}
RESUMEN
Los habitantes de las localidades del medio rural se enfrentan a serios problemas para abastecerse de agua de buena calidad. La dispersión de las viviendas complica el suministro de agua entubada y, cuando esto se logra, la administración deficiente de la infraestructura impide que los usuarios cuenten con el servicio en sus hogares de forma constante. La topografía irregular es otro problema que dificulta la instalación de infraestructura para abastecer a todas las viviendas. Por ello, se deben buscar otras formas de suministro, como la captación de agua de lluvia. La calidad del agua de lluvia es aceptable para uso doméstico y agrícola, pero no para el consumo. El objetivo de esta investigación fue validar un prototipo de sistema de captación de agua de lluvia desarrollado en el Laboratorio de Tecnologías Hídricas Innovadoras de la Universidad Autónoma Chapingo. La validación estuvo enfocada en tres aspectos: el diseño del sistema, la utilización del ferrocemento como material de construcción de la cisterna y el esquema de trabajo coparticipativo entre la universidad y los usuarios. Los resultados mostraron que el diseño fue adecuado para proveer agua con dos propósitos: uso doméstico en el hogar y consumo humano. La utilización del ferrocemento funcionó de acuerdo con lo esperado en cuanto a resistencia del material y buen desempeño. El esquema de participación con trabajo al inicio provocó decepción en los usuarios, porque esperaban recibir el apoyo sin realizar ningún tipo de aportación. Al darse cuenta de que el trabajo implicaba capacitación, se generó expectativa y el esquema resultó exitoso. El beneficio inmediato y la participación directa en la construcción dieron como resultado la adopción rápida de la tecnología.
\end{abstract}

Palabras clave: dispersión de viviendas, escasez de agua, tecnologías hídricas, ecotecnología, ferrocemento

\begin{abstract}
People of rural communities have issues to supply themselves with water of good quality. The dispersion of houses complicates the supply of drinking water and, when this is achieved, poor infrastructure management does not allow users to have water on a constant basis. Irregular topography is another problem that makes it impossible to install infrastructure to supply all homes. Because of this, other forms of supply should be sought, such as rainwater harvesting. The quality of rainwater is acceptable for domestic and agricultural use, but not for consumption. The objective of this research was to validate a prototype rainwater collection system developed in the Laboratorio de Tecnologías Hidricas Innovadoras of the Universidad Autónoma Chapingo. The validation was focused on three aspects: the design of the system, the use of ferrocement as a construction material for the tank and a teamwork scheme between the university and the users. The results showed that the design was adequate to provide water for two purposes: domestic use in the home and human consumption. The use offerrocement worked in accordance with the expected in terms of material strength and good performance. The scheme of teamwork at the beginning caused disappointment in the users, because they expected to receive the support without making any type of contribution. When realizing that the work involved training, expectation was generated and the scheme was successful. The immediate benefit and direct participation in the construction resulted in the rapid adoption of the technology.
\end{abstract}

Keywords: dispersion of houses, lack of water, water technologies, ecotechnology, ferrocement

\section{Introducción y Revisión bibliográfica}

La política de suministrar agua mediante una red de agua potable para las comunidades rurales resulta insostenible (Ortiz et al., 2009). Las zonas rurales marginadas presentan asentamientos dispersos que requieren redes costosas de distribución para proveer agua a pocas viviendas. La condición de

1 Colegio de Postgraduados, Posgrado de Hidrociencias. Texcoco, Estado de México, México.

2 Universidad Autónoma Chapingo, Departamento de Irrigación. Chapingo, Estado de México, México.

3 Universidad de Guadalajara, Departamento de Producción Agrícola. Guadalajara, México.

* Autor para correspondencia: javelar@colpos.mx, juan.uar@gmail.com

Fecha de recepción: 19 noviembre, 2018.

Fecha de aceptación: 31 enero, 2019.

DOI: http://dx.doi.org/10.4067/S0718-34292019005000302. Publicado en línea: 12-junio-2019. 
recurso asociado a la vida le ha conferido al tema del suministro de agua potable una alta valoración social y ha generado una fuerte intervención de factores políticos. Los intereses de corto plazo se privilegian sobre los de largo plazo (Corrales, 2004). Además, las inversiones, que no son suficientes por el aumento poblacional, no siempre resultan exitosas por la deficiente administración de la infraestructura.

En muchos casos, a pesar de contar con instalaciones para agua entubada en las comunidades, sus habitantes se enfrentan a problemas que limitan el uso de la infraestructura y se queda en el abandono. Algunos de los problemas detectados en localidades rurales son los siguientes: mecanismos deficientes para la recaudación de cuotas que permitan el mantenimiento adecuado de la infraestructura, falta de capacitación en el uso de las bombas y mantenimiento de las instalaciones, y localización inadecuada de los tanques de distribución. Toda esta problemática en torno al suministro de agua para las comunidades hace pensar en alternativas diferentes. Una de ellas es captar el agua de lluvia.

La captación de agua de lluvia se ha utilizado en diferentes épocas y culturas, por ser un medio fácil y sensato para obtener agua para el consumo humano y para el uso agrícola (Díaz et al., 2000). También se utiliza en la recarga de acuíferos para la restauración del ciclo hidrológico (Salinas et al., 2016). Los sistemas para la captación y el aprovechamiento del agua de lluvia se han convertido en medidas amigables, económicas y ecológicas en la gestión de agua. Sin embargo, su uso no se ha generalizado debido a que los periodos de recuperación financiera son largos (Silva, et al., 2015; Şahin, et al., 2018).

La composición química del agua de lluvia presenta alta variación espacial y temporal, y es afectada por la contaminación atmosférica de las ciudades. A pesar de ello, su calidad fisicoquímica en general es aceptable (Pérez et al., 2006). No se puede decir lo mismo de su calidad sanitaria. Numerosos estudios han indicado que la calidad microbiana no cumple con las pautas establecidas para consumo humano (Dobrowsky et al., 2014; Kobayashi, 2014; León et al., 2016; Waso et al., 2018). El agua de lluvia sin tratamiento, proveniente de techos y depósitos limpios, puede usarse de manera segura para riego de cultivos y jardines, actividades de limpieza en el hogar, piscicultura y servicios sanitarios (Arboleda, 2016; Devkota et al., 2015; Ghisi et al., 2007). No se recomienda para consumo humano de manera directa. Es necesaria la potabilización mediante algún sistema de tratamiento certificado.
En México, como en otros países, se han tomado iniciativas para suministrar agua proveniente de la lluvia en comunidades rurales. Ejemplo de ello es lo que se realiza en el Laboratorio de Tecnologías Hídricas Innovadoras de la Universidad Autónoma Chapingo (LTHI). En este laboratorio se hacen pruebas con diferentes modelos de sistemas de captación de agua de lluvia a nivel domiciliario y se han mejorado varios tipos de bombas hidráulicas manuales. Una vez probadas, las tecnologías se han transferido de manera exitosa a comunidades rurales de varios estados del país.

En estos sistemas la lluvia se recolecta en los techos por dos razones: porque permite que el agua captada fluya hasta la cisterna de almacenamiento por gravedad y porque los techos se encuentran limpios o son fáciles de limpiar. Se recomienda su limpieza porque son acumuladores de contaminantes como polvo y heces de aves, por lo que es importante eliminar el agua que se capta en los primeros minutos de un evento de precipitación, utilizando algún tipo de trampa (Gutiérrez y Rubio, 2014). El agua de lluvia como fuente alterna tiene calidad adecuada para su consumo, siempre y cuando se siga, desde su captación, almacenamiento, tratamiento y disposición, normas básicas de higiene (Pérez et al., 2017).

El objetivo del estudio fue elaborar el proyecto de sistema de captación de agua de lluvia y construirlo para validar su funcionamiento, con la meta de abastecer de agua para consumo humano y uso doméstico a 11 familias de la comunidad de Los Achotes, municipio de San Luis Acatlán, en el estado de Guerrero. Resulta trascendente corroborar las ventajas del modelo de sistema de captación de agua de lluvia desarrollado en el LTHI, así como los beneficios de la técnica de construcción utilizando ferrocemento.

\section{Materiales y métodos}

El presente estudio se llevó a cabo en la localidad de Los Achotes, municipio de San Luis Acatlán Guerrero, localizada a $120 \mathrm{~m}$ de altitud y en las coordenadas $16^{\circ} 40^{\prime} 09^{\prime \prime}$ de latitud norte y 98 49'04" de longitud oeste. La comunidad, considerada como de "muy alta marginación" (según el Instituto Nacional de Estadística y Geografía), está conformada por 146 viviendas, de las cuales menos de la mitad cuenta con agua entubada y de manera intermitente. De las familias que no tienen acceso al servicio, se seleccionaron 11, las que se evaluaron como las más vulnerables. 


\section{Cálculo del potencial de captación de lluvia}

La precipitación neta $(\mathrm{PN})$ se utiliza para determinar la cantidad de lluvia real que puede ser aprovechada. También se utiliza en hidrología para estimar la lluvia que cae en el suelo, después de haber sido interceptada y aprovechada por las plantas (Flores et al., 2016).

La PN se calculó a partir de los registros disponibles de precipitación (P) en la estación climatológica más cercana. En captación de agua de lluvia, la PN se define como la cantidad de agua que permanece disponible para el sistema, después de restar las pérdidas por factores como salpicado de las gotas de lluvia, velocidad del viento, evaporación, fricción, tamaño de la gota, considerados en un coeficiente de $85 \%(0,85)$, de acuerdo con la experiencia desarrollada en el CIDECALLI-CP (Centro Internacional de Demostración y Capacitación en Aprovechamiento del Agua de Lluvia, localizado en el Colegio de Postgraduados, campus Montecillo, México).

Se utilizaron los techos como superficie para captar el agua de lluvia. El material con que están construidos desempeña un papel importante en la eficiencia de captación, porque se relaciona con la facilidad o resistencia que opone al flujo del agua. Las pérdidas de agua en los techos varían, dependiendo del material con que están construidos (Chacón, 2014).

La eficiencia de la captación del agua de lluvia depende del coeficiente de escurrimiento de los materiales del área de captación, el cual varía de 0.1 a 0.9. De acuerdo con Anaya, 2017, el coeficiente de escurrimiento (Ce) para hojas de metal acanaladas es de $0,7-0,9$, y para este proyecto se tomó el valor medio que es 0,8 .

La fórmula para estimar la precipitación neta es:

$\mathrm{PN}=\mathrm{P} \times$ ๆ captación

Donde:

ฤ captación es $=\mathrm{CE} \times 0,85$

$\mathrm{PN}=$ precipitación neta $(\mathrm{mm})$

$\mathrm{P}=$ precipitación $(\mathrm{mm})$

ๆ captación $=$ eficiencia de captación del agua de lluvia

$\mathrm{Ce}=$ coeficiente de escurrimiento

Según Anaya, 2017, cuando las precipitaciones medias mensuales son menores de $40 \mathrm{~mm}$ y de baja intensidad $\left(\mathrm{mm} \mathrm{h}^{-1}\right)$, se recomienda no considerarlas, sobre todo si se presentan durante las épocas secas, cuando la cantidad y calidad del agua de lluvia no es de consideración para su almacenamiento. A la precipitación obtenida después de quitar estos meses que tienen menos de $40 \mathrm{~mm}$ de precipitación se le denomina PNN.

\section{Socialización del proyecto}

Una de las primeras dificultades del proyecto fue seleccionar las viviendas. Alrededor de 30\% del total de viviendas tiene lámina de asbesto. Se decidió no elegir este tipo de viviendas por los problemas que ese material provoca en la salud humana (Barrera et al., 2010; García et al., 2012).

En el inicio del proyecto se realizaron recorridos y entrevistas en la comunidad para elegir 11 viviendas con mayor carencia de agua y alto grado de marginación. También se tomó en cuenta la disponibilidad y entusiasmo de la familia para aportar la mano de obra, a la cual se le capacitó y supervisó en cada etapa de construcción. Después de seleccionar las familias, se planeó la construcción del sistema, cuantificando el área, midiendo la altura del techo de cada vivienda y escogiendo el sitio idóneo para construir cada componente.

\section{Características del sistema construido}

El modelo de captación y aprovechamiento de agua de lluvia que se construyó en esta comunidad tiene los siguientes componentes:

1. Mecanismo de captación de agua en los techos: canaleta galvanizada en forma semicircular, de $20 \mathrm{~cm}$ de diámetro y $15 \mathrm{~cm}$ de profundidad.

2. Filtro de arena. Caja construida con tabiques, rellena de arena gruesa lavada, de $40 \mathrm{~cm}$ de ancho, $60 \mathrm{~cm}$ de largo y $40 \mathrm{~cm}$ de profundidad. Su función es retener sólidos del agua proveniente de los techos.

3. Cisterna. Estructura cilíndrica con capacidad efectiva de $11 \mathrm{~m}^{3}$, construida con ferrocemento. Diámetro de 2,5 $\mathrm{m}$ y profundidad de $2,5 \mathrm{~m}$.

4. Bomba hidráulica manual. Mecanismo construido con tubos de PVC, pistón de hule y estructura metálica, que al accionar una palanca permite la extracción del agua hasta una altura máxima de $12 \mathrm{~m}$. En este sistema se eleva el agua $5 \mathrm{~m}$ desde el fondo de la cisterna hasta la parte superior del tanque distribuidor.

5. Estructura de soporte. Es una estructura de tabiques construida sobre la cisterna para cargar 
el tinaco, el filtro purificador y al mismo tiempo proteger a la bomba manual.

6. Tanque distribuidor. Tinaco con capacidad de 2501 , colocado sobre la estructura de soporte. Su función es distribuir el agua extraída de la cisterna al lavadero y al filtro purificador.

7. Filtro purificador. Sistema de purificación por gravedad conformado por dos botes de plástico, un filtro de la marca British Berkeled $\circledast$ y una llave dispensadora de agua. El bote superior contiene el filtro y el inferior guarda el agua purificada.

8. Lavadero. Estructura prefabricada de granito con medidas de $90 \times 91 \mathrm{~cm}$, que se colocó sobre una base construida con tabiques, a una altura de $60 \mathrm{~cm}$. Incluye pileta de 701 de capacidad.

9. Pozo de absorción de aguas grises. Se construyó realizando una excavación de $1 \mathrm{~m}^{3}$, que se rellenó con grava y rocas de $15 \mathrm{~cm}$ de diámetro en promedio. Su función es filtrar las aguas grises provenientes del lavadero, para evitar la contaminación y la proliferación de mosquitos portadores de enfermedades como el dengue y chicungunya.

10. Techo. Con el fin de proporcionar sombra a los usuarios, que son principalmente las amas de casa, se colocó una estructura con láminas galvanizadas, de $2 \times 3 \mathrm{~m}$.
11. Plataforma de operación. Se construyó un piso de $3 \times 3 \mathrm{~m}$ sobre el que se colocó el lavadero.

La numeración de los componentes del sistema sigue la secuencia del flujo del agua desde la captación en el techo, hasta el filtro purificador y el filtrado de aguas grises, dependiendo del uso (Figura 1).

\section{Procedimiento de construcción}

Para llevar a cabo la ejecución del proyecto, se utilizó un modelo de colaboración participativa, en el que los beneficiados aportaron la mano de obra y la Universidad Autónoma Chapingo otorgó los materiales de construcción y la capacitación permanente.

En el desarrollo de este proyecto se capacitó a las familias en el proceso de construcción de un sistema para captar agua de lluvia. Por ello, se utilizaron técnicas de construcción que no representan un alto grado de dificultad técnica.

La técnica de construir con ferrocemento fue de fácil implementación. Las cisternas de ferrocemento son económicas y pueden ser construidas por casi todos los integrantes de la familia. Para ello se usan dos componentes principales: fierro y cemento. Su estructura de malla electrosoldada y malla gallinera tejida, y mezcla de cemento y

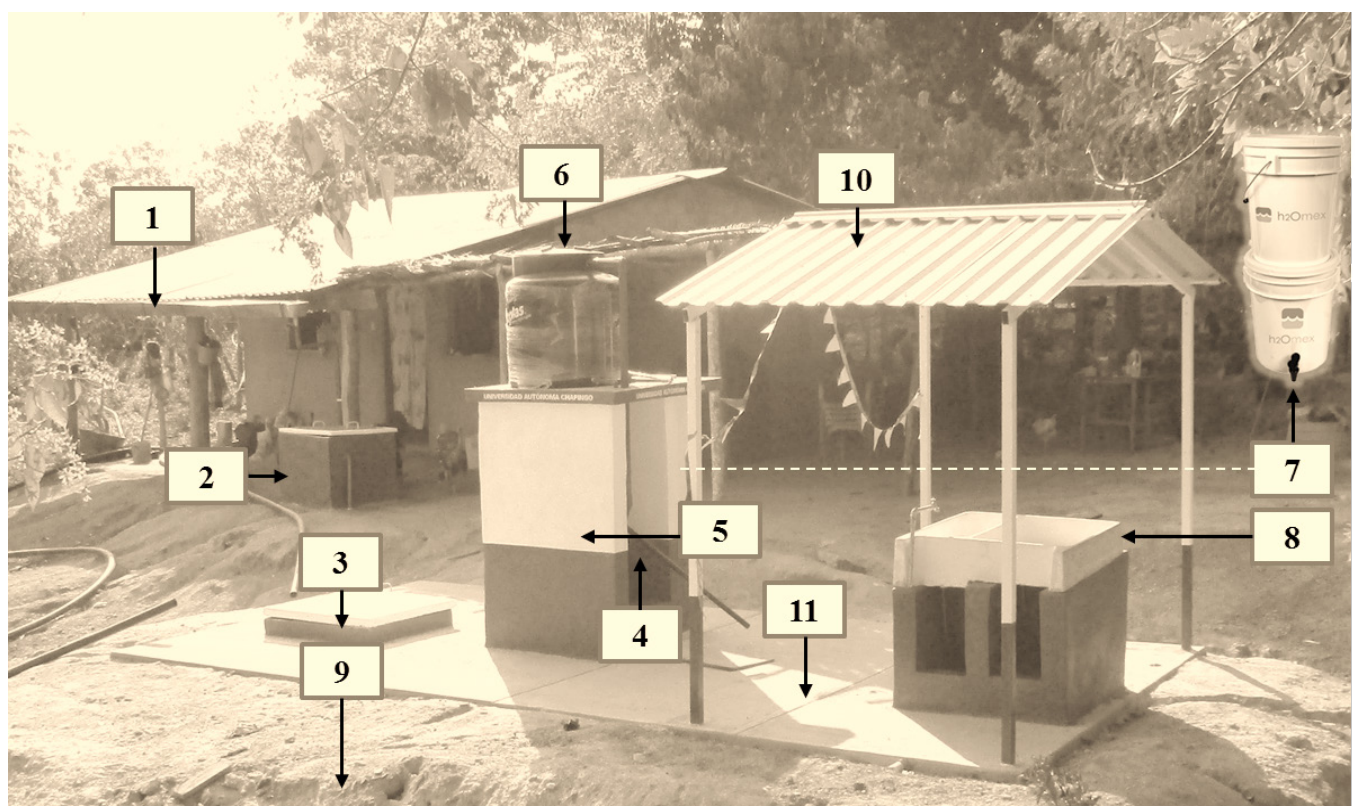

Figura 1. Componentes del sistema de captación y aprovechamiento del agua de lluvia 
arena ofrecen estabilidad, resistencia y durabilidad (Ruiz et al., 2006).

Las construcciones con ferrocemento presentan alta resistencia, baja fragilidad sísmica y sostenibilidad ambiental (Bedolla et al., 2010). Caballero Aquino, 2006, señala lo siguiente: "El ferrocemento presenta ventajas en la construcción de estructuras especiales de espesor pequeño, donde la geometría le otorga una resistencia y rigidez adecuadas. Al no emplear grava, la mezcla de arena-cemento con una consistencia "pastosa", puede adherirse al entramado de mallas embarrándola sin el uso de cimbra o encofrado [...]".

Para la construcción de la cisterna, las familias armaron la estructura metálica de soporte, tejiendo malla electrosoldada con mallas de gallinero. Después, se colocaron capas de mortero, de forma manual, en el entramado de mallas. Se aplanaron y pulieron las paredes, logrando un espesor mínimo de los muros de la cisterna de entre 8 y $10 \mathrm{~cm}$. Por último, se construyó la losa de la cisterna con un espesor de 10 cm y una tapa para el mantenimiento de $60 \times 60 \mathrm{~cm}$.

El resto de los componentes del sistema también fueron construidos por los beneficiados, bajo la supervisión permanente, capacitación y colaboración de personal especializado.

\section{Potabilización del agua de lluvia}

Para potabilizar el agua se utilizó un sistema de purificación por gravedad certificado por organismos nacionales e internacionales, que consiste en dos recipientes de plástico de grado alimenticio, un filtro de la marca British Berkeled ${ }^{\circledR}$ y una llave de paso. El sistema se colocó en la estructura de soporte y se alimenta del tinaco distribuidor. La altura de la llave para el suministro de agua potable proveniente del purificador es de $60 \mathrm{~cm}$, la cual se considera apropiada para mantenerla limpia.

\section{Resultados y discusión}

Para el presente estudio se determinó la precipitación neta (PN) que puede captarse en las viviendas seleccionadas, utilizando datos de la estación climatológica 12173 Marquelia, ubicada a 10 $\mathrm{km}$ de distancia de Los Achotes.

En la Tabla 1 se observa que los meses que superan una PN de $40 \mathrm{~mm}$ son mayo, junio, julio, agosto, septiembre y octubre.

El valor de PNN utilizado para la localidad de Los Achotes es de 939,8 mm de lluvia anuales. Esto significa que, por cada metro cuadrado de superficie de captación, se pueden aprovechar 939,8 1 cada año. Si en cada vivienda se tiene en promedio un techo de $50 \mathrm{~m}^{2}$, se cuenta con un potencial de captación y aprovechamiento de 46,99 $\mathrm{m}^{3}$ de agua cada año por familia.

Como estaba previsto, la cisterna alcanzó el $100 \%$ de su capacidad de almacenamiento durante los meses de mayo y junio, que son los dos primeros en superar los $40 \mathrm{~mm}$ de lluvia mensuales. En general, cada componente del sistema funcionó de manera adecuada, aunque fue necesario realizar reparaciones menores en las canaletas, por lluvias atípicas.

Según Imteaz et al., 2011, una cisterna de $10 \mathrm{~m}^{3}$ puede representar ahorros de agua de 25 a $144 \mathrm{~m}^{3}$, dependiendo del área de captación y del número de ocupantes de la vivienda. La superficie de los techos no es suficiente para captar el agua que una familia requiere durante todo el año, pero representa un ahorro importante para los hogares. En este proyecto no existe la posibilidad de incrementar el área de captación, por ello, el sistema está diseñado para aprovechar el agua de lluvia durante los meses de mayo a noviembre, y proporciona la infraestructura que puede ser aprovechada almacenando agua de otras fuentes durante los meses sin lluvia.

La solidez de las cisternas de ferrocemento disipó las dudas que los usuarios tenían al inicio de la construcción. Por su forma y flexibilidad son más resistentes a los sismos, si se compara con las cisternas tradicionales que se construyen con tabiques de concreto.

Al principio, los filtros proporcionados para la potabilización fueron aceptados y utilizados por 8 de las 11 familias beneficiadas. Las tres familias que no mostraron confianza en los dispositivos suministrados

Tabla 1. Precipitación neta (PN) en la estación 12173 Marquelia

\begin{tabular}{lccccccccccccr}
\hline Mes & Ene & Feb & Mar & Abr & May & Jun & Jul & Ago & Sep & Oct & Nov & Dic & Total \\
\hline Precipitación & 5,9 & 2,1 & 0,5 & 0,8 & 61,7 & 293,0 & 212.1 & 316,8 & 326,0 & 172,4 & 19,0 & 6,0 & $\mathbf{1 . 4 1 6 , 3}$ \\
PN & 4,0 & 1,4 & 0,3 & 0,5 & 42,0 & 199,2 & 144,2 & 215,4 & 221,7 & 117,2 & 12,9 & 4,1 & $\mathbf{9 6 3 , 1}$ \\
PNN ( $\geq 40 \mathrm{~mm})$ & & & & & 42,0 & 199,2 & 144,2 & 215.4 & 221.7 & 117.2 & & & $\mathbf{9 3 9 , 8}$ \\
\hline
\end{tabular}


prefirieron continuar la compra de garrafones de agua purificada, pero utilizaban el sistema de captación para el uso doméstico. Después de tres meses, todas las familias beneficiadas utilizaron los filtros proporcionados.

El impacto en la calidad de vida de las familias por la disponibilidad de agua en las viviendas se evidenció en los siguientes aspectos: ahorro promedio de \$220,00 mensuales por la adquisición de agua purificada, ahorro de tiempo en traslados al río más cercano para lavar la ropa y comodidad en las actividades domésticas que requieren el uso de agua. Un beneficio adicional lo proporcionó el pozo de absorción, el cual evita la proliferación de criaderos de mosquitos portadores de enfermedades (como el dengue y chikungunya) en los vertidos de los lavaderos.

También se obtuvieron los siguientes beneficios para el medio ambiente: 1) se disminuyó la contaminación de los cuerpos de agua por el uso de detergentes, 2) las bombas hidráulicas manuales evitaron el uso adicional de energía eléctrica, 3) el filtrado de aguas grises redujo la contaminación del agua superficial y subterránea, 4) la posibilidad de tener agua purificada en casa aminoró la compra de recipientes plásticos como garrafones y botellas de agua.

\section{Conclusiones}

Este trabajo cumplió con el objetivo principal de abastecer de agua para uso doméstico y consumo humano, a 11 familias marginadas del municipio de San Luis Acatlán, Guerrero. La aceptación del diseño entre los usuarios fue alta porque proporciona comodidad para el uso y el mantenimiento, versatilidad en su funcionamiento y facilidad de construcción. El prototipo evaluado requiere una superficie total de 18 $\mathrm{m}^{2}$. Su aplicación en viviendas del medio rural fue exitosa, pero no lo sería en zonas urbanas, donde la mayoría de las familias tiene superficies limitadas de suelo para construcción. Un aspecto que se recomienda mejorar en el diseño es incrementar la superficie del techo para sombra y utilizarla como superficie de captación.

Se capacitó a familias del medio rural en la construcción de sistemas de captación de agua de lluvia, utilizando materiales de fácil adquisición en su comunidad y aplicando técnicas sencillas de construcción. El trabajo y esfuerzo de las familias durante el proceso fue recompensado inmediatamente después de la construcción.

Aunque la captación de agua de lluvia ha ido cobrando importancia, la generalización de la tecnología, sobre todo en el medio rural, depende de las políticas públicas que los gobiernos implementen. Los usuarios no tienen la capacidad económica o no están dispuestos a invertir en estos sistemas, porque en la mayoría de los casos no son económicamente viables. La razón principal de su implementación es satisfacer el derecho humano al agua, no se busca un beneficio económico.

\section{Agradecimientos}

El autor agradece a la Universidad Autónoma Chapingo por permitirle participar como consultor externo del proyecto. También agradece al personal del Laboratorio de Tecnologías Hídricas Innovadoras por su colaboración en los trabajos de campo.

\section{Literatura citada}

Anaya, G. M.

2006. Manual del participante. In: I diplomado internacional sobre aprovechamiento del agua de lluvia. Colegio de Postgraduados. Montecillos, Estado de México. 350 p.

Arboleda, M.

2016. Evaluación de alternativas tecnológicas para el tratamiento básico del agua lluvia de uso doméstico en el consejo comunitario de la comunidad negra de Los Lagos, Buenaventura. Scientia et Technica, 21: 278-285.

Barrera, R.; Chavarría, J.; Morales, J.

2010. Mesotelioma maligno: experiencia clínico-patológica de 247 casos. Revista chilena de enfermedades respiratorias, 26 : 134-140.
Bedoya, D.; Hurtado, J.; Pujades, L.

2010. Experimental and analytical research on seismic vulnerability of low-cost ferrocement dwelling houses. Structure \& Infrastructure Engineering 6: 55-62.

Caballero Aquino, $\mathrm{T}$.

2006. Captación de agua de lluvia y almacenamiento en tanques de ferrocemento. Instituto Politécnico Nacional. México.125 p. Chacón, J.,E.; Valdés V., M.; Anaya G., D.; Gutiérrez D.; Asanza, M.; Martínez M.

2014. Diseño de sistemas de captación del agua de lluvia para producción de pepino persa (Cucumis sativus L.) bajo invernadero en Michoacán (México). UT Ciencia: Ciencia y tecnología al Servicio del Pueblo 1: 6-19. 
Corrales, M. E.

2004. Gobernabilidad de los servicios de agua potable y saneamiento en América Latina. REGA - Revista de Gestão de Água da América Latina 1: 47-58.

Devkota, J.; Schlachter, H.; Apul, D.

2015. Life cycle based evaluation of harvested rainwater use in toilets and for irrigation. J. Clean. Prod. 95: 311-321.

Díaz, D., García, P.; Solís, M.

2000. Abastecimiento de agua potable para pequeñas comunidades rurales por medio de un sistema de colección de lluvia y planta potabilizadora. Ciencia Ergo Sum, 7: 129-134.

Dobrowsky, P.H.; De Kwaadsteniet, M.; Cloete, T.E.; Khan, W. 2014. Distribution of indigenous bacterial pathogens and potential pathogens associated with roof-harvested rainwater. Appl. Environ. Microbiol, 80: 2307-2316.

Flores A., E.; Guerra D.V.; G. Terrazas G.

2016. Intercepción de lluvia en bosques de montaña en la cuenca del río Texcoco, México. Revista Mexicana de Ciencias Forestales, 7: 65-76.

García G., M.; Menéndez N., A.; Castañeda L., R.

2012. Incidencia en España de la asbestosis y otras enfermedades pulmonares benignas debidas al amianto durante el período 1962-2010. Revista española de salud pública 86: 613-625.

Ghisi, E.; Bressan, D.; Martini, M.

2007. Rainwater tank capacity and potential for potable water savings by using rainwater in the residential sector of southeastern Brazil. Build. Environ. 42: 1654-1666.

Gutiérrez, M.; Rubio-Arias, H.O.

2014. Captación pluvial en Chihuahua: una alternativa sustentable. TECNOCIENCIA Chihuahua, 8: 1-6.

Imteaz M.; Ahsan, A.; Naser, J.; Rahman A.

2011. Reliability analysis of rainwater tanks in Melbourne using daily water balance model. Resour. Conserv. Recycl. 56: 80-86.

Kobayashi M.; Oana, K.;Kawakami, Y.

2014. Incidence of Legionella and heterotrophic bacteria in household rainwater tanks in Azumino, Nagano prefecture, Japan. Microbiol Immunol. 58: 15-21.
León A., A.; Córdoba R., J.C.; Carreño S., U.F.

2016. Revisión del estado de arte en captación y aprovechamiento de agua de lluvias en zonas urbanas y aeropuertos. Revista Tecnura, 20: 141-153.

Ortiz-Guzmán, M.; Morales-Domínguez, V.; Aragón-Sulik, M. 2009. Cosecha de agua de lluvia con tanques de mampostería. Caso: San Miguel Piedras, Nochixtlán, Oaxaca. Ingeniería Hidráulica y Ambiental, 30: 25-29.

Pérez Suárez, M.; Cetina Alcalá, V. M.; Aldrete, A.; Fenn, M.

E.; Landois Palencia, L. L.

2006. Química de la precipitación pluvial en dos bosques de la cuenca de la Ciudad de México. Agrociencia, 40: 239-248.

Pérez, H., A.; O. Palacios, V.; Anaya, G., M.; Tovar, S.,J.

2017. Agua de lluvia para consumo humano y uso doméstico en San Miguel Tulancingo, Oaxaca. Revista Mexicana de Ciencias Agrícolas, 8: 1427-1432.

Ruiz, J.; Trejo, R.; Pedroza, A.; Vargas, A.

2006. Análisis del beneficio-costo en la captación agua de lluvia en el CAIS-URUZA-Uach, México. Revista Chapingo Serie Zonas Áridas, 5: 173-178.

Şahin N.; Manioğlu, G.

2018. Water conservation through rainwater harvesting using different building forms in different climatic regions. Sustainable Cities and Society, 44: 367-377.

Salinas J.; Cavazos, G.R; Vera A., J.

2016. Evaluación de un sistema de captación de agua de lluvia en la zona metropolitana de Monterrey, para su aprovechamiento como medio alternativo. Ingeniería-Revista Académica de la Facultad de Ingeniería, Universidad Autónoma de Yucatán, 20: 1-13.

Silva, C. M.; Sousa, V.; Carvalho, N. V.

2015. Evaluation of rainwater harvesting in Portugal: Application to single-family residences. Resources, Conservation and Recycling, 94: 21-34.

Waso, M.; Khan, S.; Khan, W.

2018. Microbial source tracking markers associated with domestic rainwater harvesting systems: Correlation to indicator organisms. Environmental Research, 161: 446-455. 
